\title{
SYMMETRY ANALYSIS OF A CANONICAL MEMS MODEL*
}

\author{
J. REGAN BECKHAM ${ }^{\dagger}$ AND JOHN A. PELESKO
}

\begin{abstract}
A canonical model arising in MEMS and modeling the electrostatic deflections of an elastic membrane with spatially varying dielectric properties is considered. Symmetry methods are used to determine the form of the spatially varying dielectric properties that allow for invariance under a one-parameter Lie group of transformations. In these cases it is shown how symmetry may be used to analyze the boundary value problem and construct a bifurcation diagram for the system. This bifurcation diagram relates back to the MEMS model; a non-dimensional version of the applied voltage is plotted versus the maximum deflection of the membrane.
\end{abstract}

Key words. MEMS, microelectromechanical system, Lie symmetry, Semi-linear elliptic equation.

AMS subject classifications. 34,74

1. Introduction. In the study of electrostatically actuated MEMS devices, the semi-linear elliptic equation

$$
\begin{array}{ccrlrl}
\triangle u & =\frac{\lambda}{u^{2}} & \text { in } & \Omega \\
u & =1 \quad \text { on } & \partial \Omega
\end{array}
$$

has been the subject of much analysis, [3], [5], [4], [11]. This equation models the deflection of an elastic membrane under the influence of an applied electric field. Here, $u$ represents the position of the membrane and $\lambda$ is a dimensionless parameter proportional to the applied voltage. The equation essentially says that in the steady-state, elastic forces and electrostatic forces must balance. The term on the left represents the elastic forces, while the term on the right represents the electrostatic forces. Recently, a generalized version of this equation where the dielectric properties of the membrane are allowed to vary in space has been derived, [10]. This has the effect of introducing a known function of space multiplying the term on the right hand side of Equation (1). Of interest in applications is the effect of this spatially varying term on the structure of the solution set of Equation (1).

Here, we study the one-dimensional version of this generalized equation, i.e.,

$$
\frac{d^{2} u}{d x^{2}}=\frac{\lambda f(x)}{u^{2}}
$$

with

$$
u(-1 / 2)=1 \quad \text { and } \quad u(1 / 2)=1 .
$$

The function $f(x)$ appearing on the right hand side is a dimensionless version of the spatially varying dielectric properties of the membrane. In [10] a power law profile for the dielectric property was considered, i.e., $f(x)=x^{\alpha}$. A symmetry of the differential equation was used in transforming the boundary value problem to an

\footnotetext{
*Received July 17, 2008; accepted for publication October 21, 2008.

${ }^{\dagger}$ Department of Mathematics, The University of Texas at Tyler, Tyler, TX 75799, USA (rbeckham @uttyler.edu).

${ }^{\ddagger}$ Department of Mathematical Sciences, University of Delaware, Newark, DE 19713, USA (pe lesko@math.udel.edu).
} 
initial value problem. Then, that problem was analyzed in terms of its bifurcation diagram. The symmetry of the problem is a direct result of the functional form chosen. This naturally raises the question: Which functions give rise to a symmetry? Also, can a similar analysis be performed, as in [10], regardless of the symmetry type?

We assume $f(x)$ is symmetric about the origin. With this restriction we can replace the condition $u(-1 / 2)=1$ with the condition $u^{\prime}(0)=0$. We have now reduced our problem to the interval $(0,1 / 2)$ defined by Equation (3) and boundary conditions

$$
\frac{d u}{d x}(0)=0 \quad \text { and } \quad u(1 / 2)=1
$$

Due to the boundary conditions of the problem and the domain in question, there is a possibility that functions exist which give rise to a symmetry of the equation but are not physically relevant. Also, the particular function in [10] gives rise to a scaling symmetry in both the independent and dependent variables. Here we explore how the analysis and use of this method changes as the symmetries of the problem change.

The paper is organized as follows. In Section 2 we perform a symmetry analysis of 3 to determine symmetries of the differential equation and their relationship to the functional form of $f(x)$. In Section 3 we give a necessary condition on $f(x)$ to solve the boundary value problem. In Section 4 we perform an analysis of the problem for two particular functions which arise from the symmetries found in Section 2. For each we use the symmetry to convert the boundary value problem to an initial value problem and then construct the bifurcation diagram for the problem in terms of the parameter $\lambda$ and the maximum deflection of the membrane. We further analyze the properties of the bifurcation diagram using Taylor series and phase plane techniques. Finally, in Section 5, we discuss the findings of our paper and the physical significance of the results. We relate the maximum stable deflection and maximum stable voltage to the dielectric profile of the membrane and show how it changes as the dielectric properties change.

2. Symmetry Analysis. We begin with a classical symmetry analysis of Equation (3). That is, we seek a one-parameter group of transformations,

$$
\begin{aligned}
& x^{*}=h(x, u, \epsilon) \\
& u^{*}=g(x, u, \epsilon),
\end{aligned}
$$

that leaves Equation (3) invariant. Here $f(x, u, \epsilon)$ and $g(x, u, \epsilon)$ are called the global form of the group. Considering an expansion of $x^{*}$ and $u^{*}$ about $\epsilon=0$ gives

$$
\begin{aligned}
& x^{*}=x+\epsilon\left(\frac{d x^{*}}{d \epsilon}\right)_{\epsilon=0}+\mathbf{O}\left(\epsilon^{2}\right) \\
& u^{*}=u+\epsilon\left(\frac{d u^{*}}{d \epsilon}\right)_{\epsilon=0}+\mathbf{O}\left(\epsilon^{2}\right)
\end{aligned}
$$

which is referred to as the infinitesimal form of the group. Keeping with standard notation we define

$$
\begin{aligned}
& \xi(x, u)=\left(\frac{d x^{*}}{d \epsilon}\right)_{\epsilon=0} \\
& \eta(x, u)=\left(\frac{d u^{*}}{d \epsilon}\right)_{\epsilon=0} .
\end{aligned}
$$


In finding the transformations that leave Equation (3) invariant, one needs only to find the functions $\xi(x, u)$ and $\eta(x, u)$. This is done by substitution of the above infinitesimal form of the transformations $x^{*}$ and $u^{*}$ into the differential equation and then requiring that the $\mathbf{O}(\epsilon)$ terms vanish. We begin by noting

$$
\frac{d^{2} u^{*}}{d x^{* 2}}=\frac{d}{d x}\left(\frac{d u^{*}}{d x^{*}}\right) \frac{d x}{d x^{*}}
$$

where

$$
\frac{d u^{*}}{d x^{*}}=\frac{d u}{d x}+\epsilon\left(\frac{\partial \eta}{\partial x}+\left(\frac{\partial \eta}{\partial u}-\frac{\partial \xi}{\partial x}\right) \frac{d u}{d x}-\frac{\partial \xi}{\partial u}\left(\frac{d u}{d x}\right)^{2}\right)+\mathbf{O}\left(\epsilon^{2}\right) .
$$

Using the common notation

$$
\begin{aligned}
& z=\frac{d u}{d x} \\
& \pi=\left(\frac{\partial \eta}{\partial x}+\left(\frac{\partial \eta}{\partial u}-\frac{\partial \xi}{\partial x}\right) \frac{d u}{d x}-\frac{\partial \xi}{\partial u}\left(\frac{d u}{d x}\right)^{2}\right),
\end{aligned}
$$

our governing equation becomes

$$
\begin{gathered}
\frac{d^{2} u}{d x^{2}}+\epsilon\left(\left(\frac{\partial \pi}{\partial x}+\frac{\partial \pi}{\partial u} z+\frac{\partial \pi}{\partial z} \frac{d^{2} u}{d x^{2}}\right)-\left(\frac{\partial \xi}{\partial x}+\frac{\partial \xi}{\partial u} z\right) \frac{d^{2} u}{d x^{2}}\right)+\mathbf{O}\left(\epsilon^{2}\right) \\
=\frac{\lambda}{u^{2}}+\epsilon\left(\xi \frac{\lambda f^{\prime}(x)}{u^{2}}-2 \eta \frac{\lambda f(x)}{u^{3}}\right)+\mathbf{O}\left(\epsilon^{2}\right)
\end{gathered}
$$

If we are to have a valid invariant transformation then the $\mathbf{O}(\epsilon)$ terms must vanish. That is,

$$
\left(\frac{\partial \pi}{\partial x}+\frac{\partial \pi}{\partial u} z+\frac{\partial \pi}{\partial z} \frac{d^{2} u}{d x^{2}}\right)-\left(\frac{\partial \xi}{\partial x}+\frac{\partial \xi}{\partial u} z\right) \frac{d^{2} u}{d x^{2}}=\xi \frac{\lambda f^{\prime}(x)}{u^{2}}-2 \eta \frac{\lambda}{u^{3}} .
$$

Expanding the left hand side, substituting $\frac{d^{2} u}{d x^{2}}=\lambda / u^{2}$, and collecting powers of $z$ gives

$$
\begin{aligned}
& \left(\frac{\partial^{2} \eta}{\partial x^{2}}+\left(\frac{\partial \eta}{\partial u}-2 \frac{\partial \xi}{\partial x}\right) \frac{\lambda f(x)}{u^{2}}-\xi \frac{\lambda f^{\prime}(x)}{u^{2}}+2 \eta \frac{\lambda f(x)}{u^{3}}\right) \\
& +\left(2 \frac{\partial^{2} \eta}{\partial x \partial u}-\frac{\partial^{2} \xi}{\partial x^{2}}-3 \frac{\lambda f(x)}{u^{2}} \frac{\partial \xi}{\partial u}\right) z \\
& +\left(\frac{\partial^{2} \eta}{\partial u^{2}}-2 \frac{\partial^{2} \xi}{\partial x \partial u}\right) z^{2}-\frac{\partial^{2} \xi}{\partial u^{2}} z^{3}=0
\end{aligned}
$$

This gives the following system of equations for $\xi$ and $\eta$.

$$
\begin{aligned}
& \frac{\partial^{2} \eta}{\partial x^{2}}+\left(\frac{\partial \eta}{\partial u}-2 \frac{\partial \xi}{\partial x}\right) \frac{\lambda f(x)}{u^{2}}-\xi \frac{\lambda f^{\prime}(x)}{u^{2}}+2 \eta \frac{\lambda f(x)}{u^{3}}=0 \\
& 2 \frac{\partial^{2} \eta}{\partial x \partial u}-\frac{\partial^{2} \xi}{\partial x^{2}}-3 \frac{\lambda f(x)}{u^{2}} \frac{\partial \xi}{\partial u}=0 \\
& \frac{\partial^{2} \eta}{\partial u^{2}}-2 \frac{\partial^{2} \xi}{\partial x \partial u}=0 \\
& \frac{\partial^{2} \xi}{\partial u^{2}}=0
\end{aligned}
$$


From the last two equations we have

$$
\begin{aligned}
& \xi(x, u)=g_{1}(x) \\
& \eta(x, u)=h_{1}(x) u+h_{2}(x)
\end{aligned}
$$

Making this substitution into the above system and solving gives

$$
\begin{aligned}
& \xi(x, u)=a x^{2}+b x+c \\
& \eta(x, u)=(a x+d) u,
\end{aligned}
$$

where $a, b, c$, and $d$ are arbitrary constants and $f(x)$ satisfies the ordinary differential equation

$$
f^{\prime}(x)\left(a x^{2}+b x+c\right)+f(x)(a x+2 b-3 d)=0 .
$$

This agrees with the results found in Chapter 5 of [6]. By taking different combinations of constants, and solving Equation (8), we obtain the different forms of $f(x)$ which give the corresponding symmetry. Let us first relate the infinitesimals $\xi(x, u)$ and $\eta(x, u)$ to the type of symmetry in the equation. The constant $c$ corresponds to a translation of the $x$ variable. The constants $b$ and $d$ correspond to scalings in $x$ and $u$ respectively. The constant $a$ corresponds to a nonstandard symmetry in the $x$ and $u$ variables.

Our goal is to see how the different types of symmetry affect the analysis of the problem and whether or not we can change the boundary value problem to an initial value problem. This idea of using the symmetry of an equation to convert a boundary value problem to an initial value problem was introduced by Toepfer, [12], in 1912 and was extended by Klamkin [7] and $\mathrm{Na}$ [8]. See Chapter 7 of [8] for a more detailed discussion. We will consider the forms of the infinitesimals, (6) and (7), along with the form of the solution to Equation (8). Using this information we make a suitable change of variables to convert our boundary value problem to an initial value problem. Bifurcation diagrams will then be constructed. The bifurcation diagrams will be in terms of $\lambda$ which represents the applied voltage, and $u_{\max }=1-u(0)$ which represents the maximal deflection of the membrane. These bifurcation diagrams will then be analyzed, with the aid of the initial value problem, using phase plane analysis and Taylor series.

3. A necessary condition for existence. Not all solutions, $f(x)$, to Equation (8) give rise to a solution of Equation (3). Here we give a necessary condition for the existence of a solution to our boundary value problem. First, we require $f(x)>0$ so that $u(x)$ is concave and has a range of $(0,1]$. From this we can see that $u(x)$ is bounded. Moreover, since $u(x)$ does not take on the value 0 , since that would mean it comes in contact with the ground plate, we can say that $1 / u^{2}$ is also bounded. Now if we consider Equation (3),

$$
\frac{d^{2} u}{d x^{2}}=\frac{\lambda f(x)}{u^{2}}
$$

then we can integrate with respect to $x$ and use $u^{\prime}(0)=0$ to obtain

$$
\frac{d u}{d x}=\int_{0}^{x} \frac{\lambda f(y)}{u^{2}} d y
$$


Upon integration again, we have

$$
u(x)-u(0)=\int_{0}^{x} \int_{0}^{z} \frac{\lambda f(y)}{u^{2}} d y d z .
$$

From the nature of $u(x)$, we know that the left hand side is bounded. However when we look at the right hand side we see that

$$
\begin{aligned}
\int_{0}^{x} \int_{0}^{z} \frac{\lambda f(y)}{u^{2}} d y d z & =\lambda \int_{0}^{x} \int_{0}^{z} \frac{f(y)}{u^{2}} d y d z \\
& \geq \lambda M \int_{0}^{x} \int_{0}^{z} f(y) d y d z .
\end{aligned}
$$

where $M$ is the lower bound on $1 / u^{2}$. From this we can see that the only way the right hand side can be bounded is if

$$
\int_{0}^{x} \int_{0}^{z} f(y) d y d z
$$

is bounded. This information will be used to determine if a given form of $f(x)$ will give rise to a solution to the boundary value problem. If Equation (9) is unbounded for some $f(x)$ then no solution to the boundary value problem (3), (5) will exist.

It is important to note here that in our particular problem $f(x)$ is a scaled version of the dielectric constant and thus satisfies

$$
0<c \leq f \leq 1 .
$$

In some cases the analysis can be carried through due to the boundedness of the above integral for our given $f(x)$ but may not satisfy the above condition for the dielectric constant.

4. Analysis. In this section we perform a detailed analysis of two separate cases which arise from Equation (8). For each case we use symmetry to convert our boundary value problem to an initial value problem. Then we use the initial value problem to analyze and construct the bifurcation diagram relating the parameter $\lambda$ to the maximal deflection of the membrane $u_{\max }$. In the first case Taylor expansions are used to describe the end behavior of the bifurcation diagram. In the second case a phase plane analysis is used to describe the end behavior of the bifurcation diagram. Next, we discuss cases where the symmetry is not effective and why, as well as, the general relationship between the symmetry and analysis technique used.

4.1. Case: $\mathbf{a}=\mathbf{b}=\mathbf{0}$. In this case, Equation (6) and Equation (7) reduce to

$$
\begin{aligned}
& \xi=c \\
& \eta=d u
\end{aligned}
$$

that is the symmetry is a stretch in the $u$ direction along with a translation of the $x$ variable. Solving Equation (8) and substituting into Equation (3) yields

$$
\frac{d^{2} u}{d x^{2}}=\lambda \frac{e^{\frac{3 d}{c} x}}{u^{2}}
$$

with the conditions

$$
\frac{d u}{d x}(0)=0 \quad \text { and } \quad u(1 / 2)=1
$$


At this point we construct the global form of the group to obtain

$$
\begin{aligned}
& x^{*}=x+c \epsilon \\
& u^{*}=e^{d \epsilon} u .
\end{aligned}
$$

We can now exploit the symmetry by letting

$$
u(x)=\gamma w(\zeta),
$$

where

$$
\zeta=x+\beta .
$$

Note that $\gamma$ and $\beta$ are constants. Substituting (13) and (14) into Equation (12), and defining $\alpha=\frac{3 d}{c}$ yields the problem

$$
\frac{d^{2} w}{d \zeta^{2}}=\frac{\lambda}{\gamma^{3}} \frac{e^{\alpha(\zeta-\beta)}}{w^{2}}
$$

with the conditions

$$
\frac{d w}{d \zeta}(\beta)=0 \quad \text { and } \quad \gamma w\left(\beta+\frac{1}{2}\right)=1 .
$$

Next, we make the assignment

$$
\frac{\lambda}{\gamma^{3}} e^{-\alpha \beta}=1 \quad \text { and } \quad \gamma=\frac{1}{w\left(\beta+\frac{1}{2}\right)} .
$$

Since our boundary condition is satisfied by setting $\gamma$ we impose the condition $w(\beta)=$ 1. This leads to the problem

$$
\frac{d^{2} w}{d \zeta^{2}}=\frac{e^{\alpha \zeta}}{w^{2}}
$$

with

$$
\frac{d w}{d \zeta}(\beta)=0 \quad \text { and } \quad w(\beta)=1 .
$$

We have now converted the boundary value problem to an initial value problem.

Now, using (15), we map out the bifurcation diagram, which is parameterized in terms of $\beta$, where $-\infty<\beta<\infty$. A plot of the bifurcation diagram is seen in Figure 1 for different values of $\alpha$. Notice that the initial conditions occur at $\beta$. Because of this our initial conditions change as our parameter changes and thus a phase plane analysis is of no use here. Since $\lambda$ and $u_{\max }$ depend on $w\left(\beta+\frac{1}{2}\right)$ we will consider a Taylor expansion of $w(\beta+x)$ about $x=0$ and then evaluate at $x=\frac{1}{2}$. From here we take our limits and determine the end behavior of the bifurcation diagram. Our Taylor expansion gives

$$
w(\beta+1 / 2)=w(\beta)+\frac{d w}{d \zeta}(\beta) \frac{1}{2}+\frac{\frac{d^{2} w}{d \zeta^{2}}(\beta)}{2 !} \frac{1}{2}^{2}+{\frac{d^{3} w}{d \zeta^{3}}(\beta)}_{3 !} \frac{1}{2}^{3}+\cdots
$$




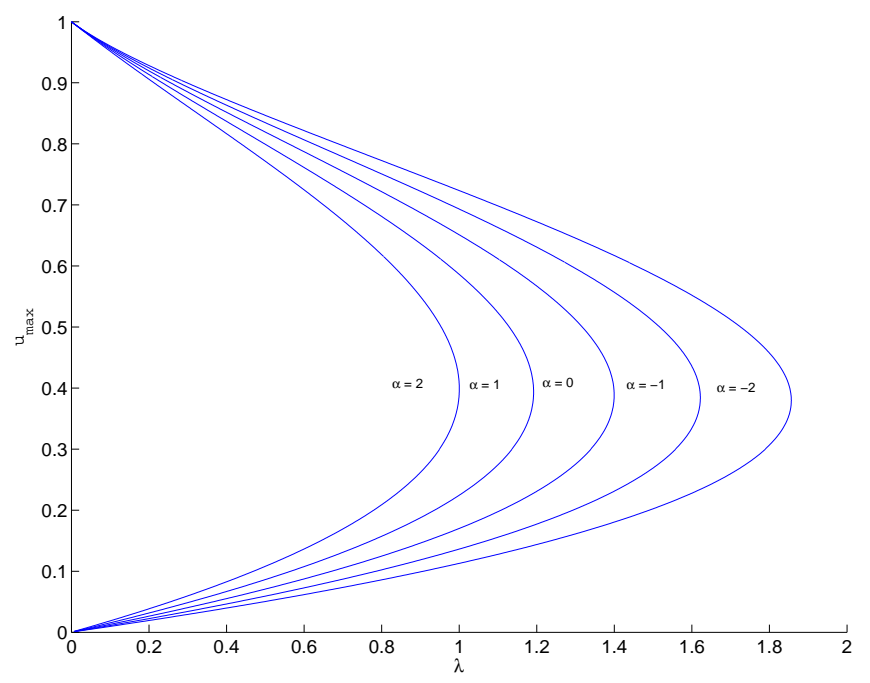

FIG. 1. Bifurcation for $-2<\alpha<2$

Substituting Equation (16), its differential consequences, and (17) into Equation (18) gives

$$
w(\beta+1 / 2)=1+0+\frac{e^{\alpha \beta}}{2 !} \frac{1}{2}^{2}+\frac{\alpha e^{\alpha \beta}-2 e^{\alpha \beta}}{3 !} \frac{1}{2}^{3}+\cdots
$$

Notice that every term in the expansion involves an $e^{\alpha \beta}$ except for the first two. Now for $\alpha<0$ we see that

$$
w(\beta+1 / 2) \rightarrow 1 \quad \text { as } \quad \beta \rightarrow \infty .
$$

This means that

$$
\lambda \rightarrow 0 \quad \text { and } \quad u_{\max } \rightarrow 0 .
$$

When $\beta \rightarrow-\infty, w\left(\beta+\frac{1}{2}\right) \rightarrow \infty$, so that

$$
\lambda \rightarrow 0 \quad \text { and } \quad u_{\max } \rightarrow 1 .
$$

When $\alpha>0$ the behavior reverses. This agrees with the bifurcation diagram in Figure 1. Note that $f(x)$ corresponds to a valid dielectric constant when $\alpha<0$.

4.2. Case: $\mathbf{b}=\mathbf{c}=\mathbf{0}$. In this case, Equation (6) and Equation (7) reduce to

$$
\begin{aligned}
& \xi=a x^{2} \\
& \eta=(a x+d) u ;
\end{aligned}
$$

that is, the symmetry is a nonstandard change in the $x$ direction along with the combination of a stretch and nonstandard change in the $u$ direction. Solving Equation (8) and substituting into Equation (3) gives

$$
\frac{d^{2} u}{d x^{2}}=\lambda \frac{e^{-\frac{3 d}{a x}}}{x u^{2}}
$$


with the conditions

$$
u^{\prime}(0)=0 \quad \text { and } \quad u(1 / 2)=1 .
$$

At this point we construct the global form of the group to obtain

$$
\begin{aligned}
x^{*} & =\frac{x}{1-\epsilon a x} \\
u^{*} & =\frac{e^{d \epsilon} u}{1-\epsilon a x}
\end{aligned}
$$

This does not suggest stretches and translations as in the previous section. However, we make an appropriate change of variables so that, in the new variables, we have stretches and translations. Let us make the assignment

$$
y=\frac{u}{x} \quad \text { and } \quad \zeta=\frac{1}{x} .
$$

Notice that this assignment is suggested by the form that $f(x)$ takes. Substituting (23) into Equation (22) gives

$$
\frac{d^{2} y}{d \zeta^{2}}=\frac{\lambda e^{\alpha \zeta}}{y^{2}}
$$

where the boundary conditions become

$$
\lim _{\zeta \rightarrow \infty}\left(\zeta y_{\zeta}-y\right)=0 \quad \text { and } \quad y(2)=2 .
$$

Then, from the global form, Equation (24) is invariant under

$$
\begin{aligned}
& y^{*}=e^{d \epsilon} y \\
& \zeta^{*}=\zeta-a \epsilon .
\end{aligned}
$$

From here we exploit our symmetry by letting

$$
y(\zeta)=\gamma w(\tau) \quad \text { where } \quad \tau=\zeta+\sigma
$$

Note $\gamma$ and $\sigma$ are constants. Substitution of (25) into Equation (24) yields

$$
\frac{d^{2} w}{d \tau^{2}}=\frac{\lambda e^{\alpha(\tau-\sigma)}}{\gamma^{3} w^{2}}
$$

with boundary conditions

$$
\lim _{\tau \rightarrow \infty}(\tau-\sigma) w_{\tau}-w=0 \quad \text { and } \quad w(2+\sigma)=\frac{2}{\gamma} .
$$

Next, we let

$$
\frac{\lambda e^{-\alpha \sigma}}{\gamma^{3}}=1 \quad \text { and } \quad \gamma=\frac{2}{w(2+\sigma)},
$$

so our problem becomes

$$
\frac{d^{2} w}{d \tau^{2}}=\frac{e^{\alpha \tau}}{w^{2}}
$$


with

$$
\lim _{\tau \rightarrow \infty}(\tau-\sigma) w_{\tau}-w=0
$$

Similar to previous cases we impose the condition

$$
\lim _{\tau \rightarrow \infty} w=1 \text {. }
$$

At this point we have converted our boundary value problem to an initial value problem.

Since our initial conditions occur at infinity, implementing a standard numerical integration method to map out the bifurcation diagram is difficult. Here we use a numerical shooting method to map out the bifurcation diagram. A plot of the diagram for various $\alpha$ is seen in Figure 2 .

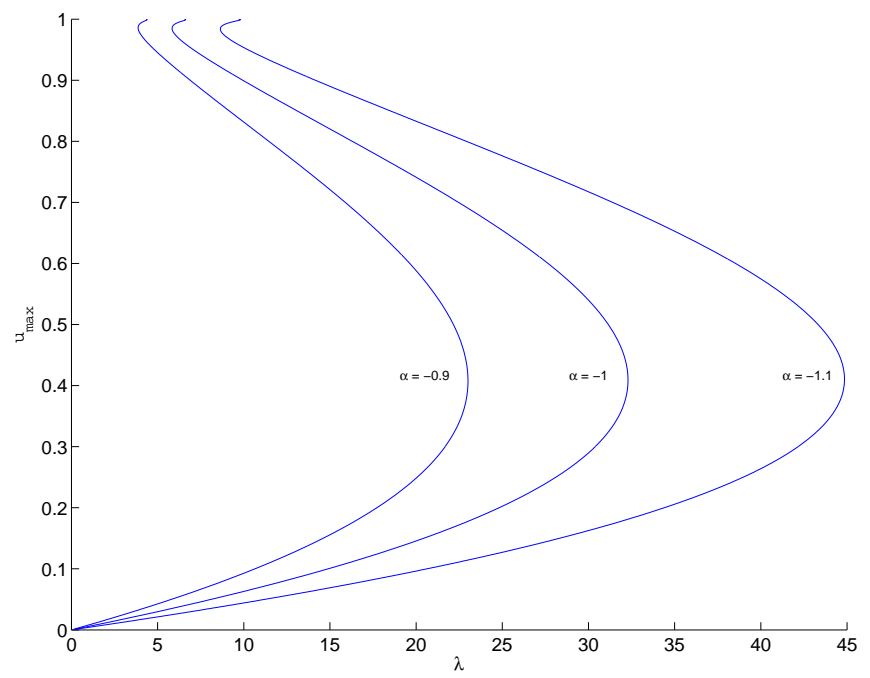

FIG. 2. Bifurcation for $\alpha<0$

Although we did not use the initial value problem to construct the bifurcation diagram, it will be beneficial in analyzing the behavior of the bifurcation diagram. Since our initial conditions do not change as the parameters change, we perform a phase plane analysis. First, we make the problem autonomous by substituting $w(\tau)=v(\tau) e^{\frac{\alpha \tau}{3}}$ into Equation (26) to obtain

$$
\frac{d^{2} v}{d \tau^{2}}+\frac{2}{3} \alpha \frac{d v}{d \tau}+\frac{\alpha^{2}}{9} v=\frac{1}{v^{2}} .
$$

Next, we convert to a first order system. Let $p=1 / v$ and $q=v^{\prime} / v$ so that Equation (31) becomes

$$
\begin{aligned}
& \frac{d p}{d \tau}=-p q \\
& \frac{d q}{d \tau}=p^{3}-\frac{2}{3} \alpha q-\frac{\alpha^{2}}{9}-q^{2} .
\end{aligned}
$$


With this change of variables, our initial conditions convert to

$$
\lim _{\tau \rightarrow \infty} p=0 \quad \text { and } \quad \lim _{\tau \rightarrow \infty} q=-\frac{\alpha}{3} .
$$

By analyzing the system given by (32) and (33), we see there are two critical points, $(p, q)$,

$$
\begin{aligned}
& P_{1}\left(0, \frac{-\alpha}{3}\right) \\
& P_{2}\left(\left(\frac{\alpha^{2}}{9}\right)^{1 / 3}, 0\right)
\end{aligned}
$$

with eigenvalues for the linearized system given by

$$
\begin{aligned}
& \mu_{1}=0, \mu_{2}=\frac{\alpha}{3} \\
& \mu_{1,2}=\frac{-\alpha}{3} \pm i \frac{\alpha \sqrt{2}}{3}
\end{aligned}
$$

respectively. From this we conclude

- $P_{1}$ is a stable node.

- $P_{2}$ is an unstable spiral.

Figure 3 give a plot of the phase plane for $\alpha=-1$. In terms of our new variables, $\lambda$

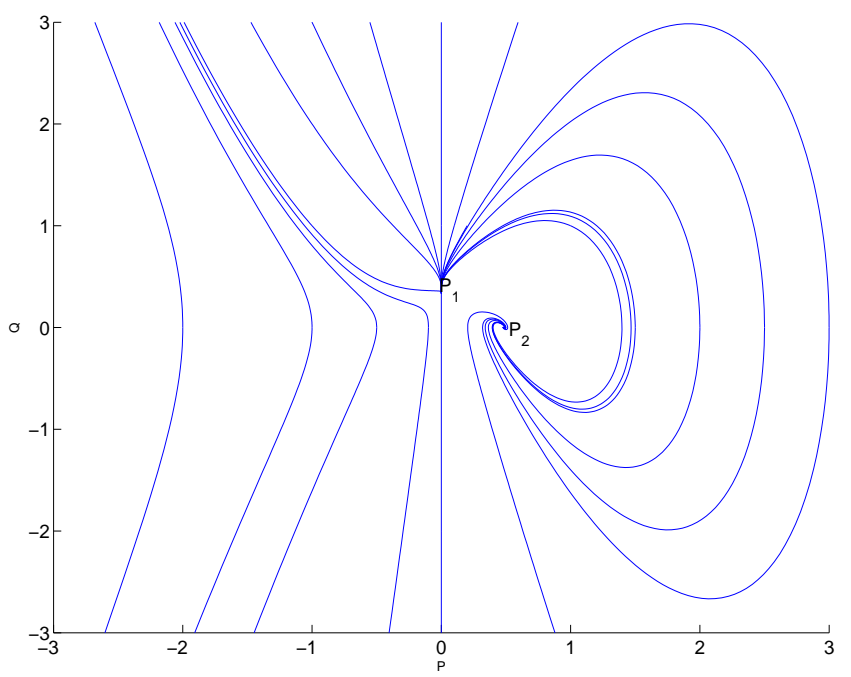

FIG. 3. Phase portrait in the $p-q$ plane when $\alpha=-1$. Here $P_{1}$ corresponds to a stable node, and $P_{2}$ corresponds to an unstable focus.

and $u_{\max }$ become

$$
\begin{aligned}
\lambda & =\frac{8 p(2+\sigma)^{3}}{e^{2 \alpha}}, \\
u_{\max } & =1-\lim _{\tau \rightarrow \infty} \frac{2 p(2+\sigma) e^{\frac{\alpha \tau}{3}}}{p(\tau) e^{\frac{\alpha}{3}(2+\sigma)}(\tau-\sigma)} .
\end{aligned}
$$


To determine the behavior of $u_{\max }$ at the ends of the bifurcation diagram, we need to determine the behavior of $p$ as $\tau \rightarrow \infty$. Now, as $\tau \rightarrow \infty$ we approach the point $P_{1}$ in the phase plane. Since $p \rightarrow 0$ implies $1 / v^{2} \rightarrow 0$, we can set the right hand side of Equation (31) equal to zero and solve for $v$. Doing this gives the solution

$$
v(\tau)=c_{1} e^{-\frac{\alpha}{3} \tau}+c_{2} \tau e^{-\frac{\alpha}{3} \tau} .
$$

Notice here that if $\tau \rightarrow \infty$, then $v \rightarrow \infty$. This is consistent with our above assumption. Substituting (37) into Equation (36) and taking the limit gives

$$
u_{\max }=1-\frac{2 c_{2} p(2+\sigma)}{e^{\frac{\alpha}{3}(2+\sigma)}} .
$$

At this point we have $\lambda$ and $u_{\max }$ parameterized in terms of $\sigma$. Now as $\sigma \rightarrow-\infty$ we approach the critical point $P_{2}$. From the linearized system we can approximate the solution around this point to conclude that

$$
\lambda \rightarrow \frac{8 \alpha^{2}}{9 e^{2 \alpha}} \quad \text { and } \quad u_{\max } \rightarrow 1 \quad \text { as } \quad \sigma \rightarrow-\infty .
$$

Note here that our bifurcation diagram is found by mapping out a trajectory in the phase plane and recall that the critical point $P_{2}$ is a spiral. Approaching the critical point $P_{2}$ corresponds to $\lambda \rightarrow \frac{8 \alpha^{2}}{9 e^{2 \alpha}}$; therefore, we have an infinite number of folds in the bifurcation diagram and an infinite number of solutions when $\lambda=\frac{8 \alpha^{2}}{9 e^{2 \alpha}}$. When $\sigma \rightarrow \infty$ we approach the critical point $P_{1}$. From this we see that

$$
\lambda \rightarrow 0 \quad \text { and } \quad u_{\max } \rightarrow 0 \quad \text { as } \quad \sigma \rightarrow \infty .
$$

This agrees with the bifurcation diagram plotted in Figure 2 .

4.3. Other cases. Now we address all cases not considered in the previous sections. If we consider the case of $a=b=c=0$ then Equation (8) has the solution

$$
f(x)=0 .
$$

Since the right hand side of Equation (3) vanishes this case is of no interest. The case of $a=b=d=0$ gives rise to $f(x)$ being a nonzero constant. For an indepth look at this case see [2]. The cases $a=c=d=0, b=c=d=0$, and $c=d=0$ give rise to the solutions

$$
f(x)=\frac{\lambda}{x^{2}}, \quad f(x)=\frac{\lambda}{x}, \quad \text { and } \quad f(x)=\frac{\lambda(a x+b)}{x^{2}}
$$

to Equation (8) respectively. From Equation (9) we see that these cases do not give rise to physically relevant solutions. The two cases, $a=d=0$ and $b=d=0$, do not correspond to any type of scaling of the $u$ variable. Because of this, the symmetry cannot be used to convert the boundary value problem to an initial value problem. A numerical shooting method can be used to construct the bifurcation diagram, however we cannot use the properties of an initial value problem to analyze the bifurcation diagram. A detailed analysis of the remaining cases can be found in [1].

Notice the dependence on the stretching of $u, d \neq 0$, in the ability to use the symmetry to convert the boundary value problem to an initial value problem. For each case considered above we used the stretching invariance of $u$ to satisfy one of our boundary conditions. If the stretching invariance was readily identifiable we made a change of variables and then used the stretching invariance of the new dependent variable to satisfy one of our boundary conditions. In each of the cases where symmetry is not used the stretching invariance of $u$ is removed, $d=0$. 
5. Discussion. In this paper our goal was to explore the use of symmetry methods to analyze the electrostatic deflections of an elastic membrane with tailored dielectric properties, as introduced in [10]. It was shown in the one-dimensional case that a number of dielectric profiles, in addition to the power law profile considered in [10], give rise to a symmetry of the differential equation. In addition, it was shown that those symmetries containing a scaling of the dependent variable, and those which can be put into this form via a change of variables, allow for the removal of a boundary condition, thus changing our boundary value problem to an initial value problem. In these cases we were able to parameterize our problem in terms of the transformation coefficients of the independent variable and then integrate the initial value problem to construct the desired bifurcation diagram. In addition, we were able to use phase plane analysis and Taylor series to further analyze the problem and confirm the end behavior of the bifurcation diagram. It was observed that without a scaling of the dependent variable, the symmetry of the equation cannot be used to convert our initial value problem to a boundary value problem. For the two-dimensional disk geometry we observed that the power law profile considered in [10] is the only dielectric profile admitting a symmetry where a physical solution is possible. This was seen by observing that the integral of our dielectric profile must be finite.

From a physical standpoint it is interesting to note that, regardless of the dielectric property of the membrane, that is the functional form of $f(x)$, the pull-in voltage was increased. In particular we consider the power law profile considered in [10] and the exponential form when $a=b=0$. For the power law profile the membrane is tailored so that the center of the membrane, which is the most unstable, is to be more of an insulator and the edges, where it is most stable, are to be closer to a perfect conductor. For the exponential profile, $f(x)=e^{\alpha x}$, the tailoring is just the opposite. In either case, the change in $\alpha$ results in a larger operating voltage; however, the maximal possible deflection is reduced for the exponential profile where as the power law profile causes the maximal possible deflection to increase.

To illustrate this, Figure 4 gives a plot of the "pull-in" voltage versus the "pullin" distance as the values of $\alpha$ are varied. The solid curve represents the power law profile with $\alpha$ increasing from zero as you move along the curve from left to right. The dashed curve represents the exponential profile with $\alpha$ decreasing form zero as one moves from left to right. Recall that $\alpha$ must be negative in the exponential profile to represent a valid dielectric constant. This says that making the center of the membrane more of an insulator will cause an increase in the "pull-in" voltage and an increase in the "pull-in" distance while making the edges of the membrane more of an insulator will increase the "pull-in" voltage while decreasing the "pull-in" distance. This relationship seems to hold true for the other profile considered in the analysis, as well as those considered in [1].

\section{REFERENCES}

[1] J. R. Beckham, Analysis of Mathematical Models of Electrostatically Deformed Elastic Bodies, Dissertation, University of Delaware, 2008.

[2] D. A. Bernstein, P. Guidotti, and J. A. Pelesko, Analytical and Numerical Analysis of Electrostatically Actuated MEMS Devices, in Proceedings of Modeling and Simulation of Microsystems, San Diego, CA, 2000, pp. 489-492.

[3] G. Flores, G. Mercado, J. A. Pelesko, and N. Smyth, Analysis of the Dynamics and Touchdown in a Model of Electrostatic MEMS, SIAM J. Appl. Math., 67:2 (2006), pp. 434446. 


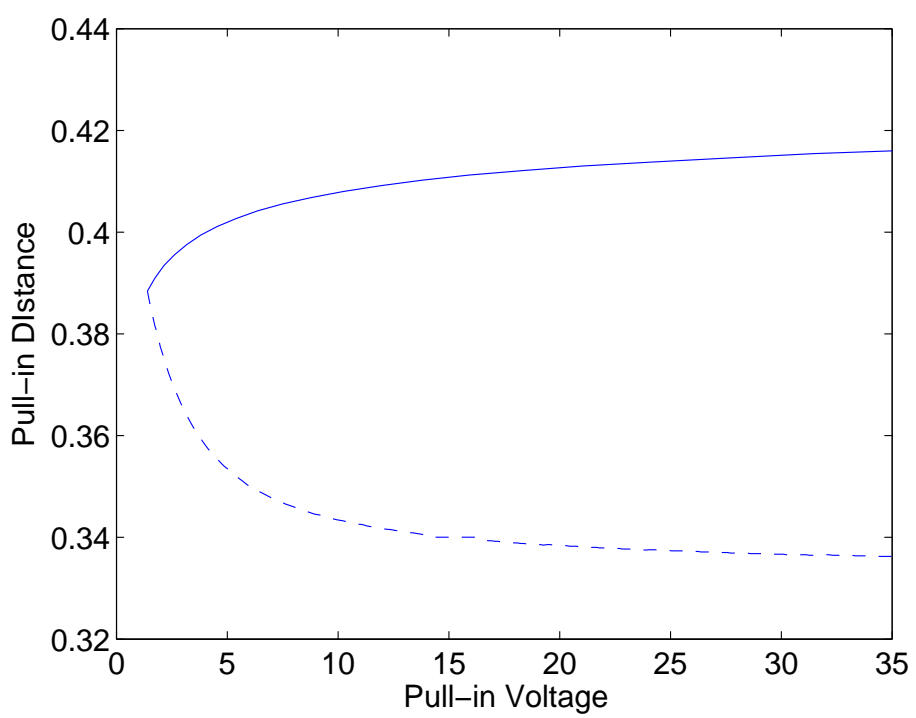

FIG. 4. Plot of the pull-in distance versus the pull-in voltage as the dielectric profile is changed. The solid curve represents the power law profile with $\alpha$ increasing from left to right. The dashed curve represents the exponential profile with $\alpha$ decreasing from left to right. The point where the two curves meet corresponds to $\alpha=0$.

[4] N. Ghoussoub And Y. Gou, On the Partial Differential Equations of Electrostatic MEMS Devices: Stationary Case, SIAM J. Math. Anal., 38:5 (2006), pp. 1423-1449.

[5] Y. Guo, Z. PAN, AND M. J. WARD, Touchdown and Pull-in Voltage Behavior of a MEMS Device with Varying Dielectric Properties, SIAM J. Appl. Math., 66:1 (2005), pp. 309338.

[6] J. M. HILl, Differential Equations and Group Methods for Scientists and Engineers, CRC Press, Boca Raton, FL, 1992.

[7] M. S. Klamkin, Transformation of boundary value problems into initial value problems, J. Math. Anal. Appl., 32, 308.

[8] T. Y. NA, Computational Methods in Engineering Boundary Value Problems, Academic Press, New York, 1979.

[9] H. C. Nathanson, W. E. Newell, R. A. Wickstrom, and J. R. Davis, The Resonant Gate Transistor, IEEE Trans. on Electron Devices, 14 (2007), pp. 117-133.

[10] J. A. Pelesko, Mathematical Modeling of Electrostatic MEMS with Tailored Dielectric Properties, SIAM J. Appl. Math., 62:3 (2002), pp. 888-908.

[11] J. A. Pelesko and D. H. Bernstein, Modeling MEMS and NEMS, Chapman \& Hall/CRC, New York, NY, 2003.

[12] K. Toepfer, Grenzschichten in Flussigkeiten mit lkeiner Reibung, Z. Math. Phys., 60 (1912). 
J. R. BECKHAM AND J. A. PELESKO 